\title{
Effects of Cross Training with Bilateral Ankle Dorsiflexor Strengthening Exercise on the Muscle Activity of the Paralytic Tibialis Anterior, Balancing Ability, and Gait Function in Patients with Chronic Stroke: A Preliminary Randomized, Controlled Study
}

\author{
Sung-Chan Park, PT, BS • Jun-Nam Ryu, PT, MS ${ }^{1} \cdot$ Jae-Man Park, PT, MS • \\ Byoung-Do Seo, PT, MS ${ }^{2} \cdot$ In-Tae Ryu, PT, PhD • Yong-Jun Cha, PT, PhD ${ }^{3 \dagger}$ \\ Department of Physical Therapy, Daejeon Rehabilitation Hospital \\ ${ }^{1}$ Department of Physical Therapy, Yeoju University \\ ${ }^{2}$ Department of Physical Therapy, Kyungwoon University \\ ${ }^{3}$ Department of Physical Therapy, College of Health and Medical Science, Dajeon University
}

Received: October 27, 2019 / Revised: October 29, 2019 / Accepted: November 4, 2019

(C) 2019 J Korean Soc Phys Med

\section{| Abstract |}

PURPOSE: This study examined the effects of bilateral ankle dorsiflexors-strengthening exercise on the paralytic tibialis anterior activity, balance ability, and gait function of patients with chronic stroke.

METHODS: Nineteen patients with chronic stroke were assigned randomly to the experimental and control groups. All participants received general physical therapy for 60-minutes per session, five times a week, for 6 weeks. In addition, the experimental group $(\mathrm{n}=9)$ performed bilateral ankle dorsiflexion muscle-strengthening training three times a week, 30 minutes per session, for six weeks. The control

†Corresponding Author : Yong-Jun Cha

cha0874@dju.kr, https://orcid.org/0000-0002-8553-7098

This is an Open Access article distributed under the terms of the Creative Commons Attribution Non-Commercial License (http://creativecommons.org/licenses/by-nc/3.0) which permits unrestricted non-commercial use, distribution, and reproduction in any medium, provided the original work is properly cited. group $(n=10)$ performed the paraplegic ankle dorsiflexion muscle- strengthening training in the same manner. Before and after the intervention, the paralytic tibialis anterior muscle activity, timed up and go test (TUG), and 10m walking test (10 MWT) were performed.

RESULTS: Both groups showed significant improvement in the post-intervention muscle activity of the paralytic tibialis anterior, TUG, and 10MWT compared to that before the intervention $(\mathrm{p}<.05)$, but the differences between the two groups were not significant ( $\mathrm{p}>.05)$.

CONCLUSION: Bilateral ankle dorsiflexors strengthening exercise is an effective cross-training method to improve the muscle activity of the paraplegic tibialis anterior, balance ability, and walking function in chronic stroke patients.

Key Words: Ankle dorsiflexor, Cross-education, Electromyography, Stroke 


\section{Introduction}

Stroke is a major adult disease and a major socioeconomic burden worldwide [1]. Representative disorders caused by stroke include poor balance and gait function [2,3]. This can lead to difficulties in daily life, which is a very important factor that reduces the quality of life of stroke patients [4]. Therefore, the major goal of rehabilitation for stroke patients is to acquire a safe and efficient gait function [5].

One of the major factors limiting gait in stroke patients is muscle weakness in the lower limbs [6]. Patients with chronic stroke show significant loss of muscle strength in both the paralyzed and non-paralyzed side compared to general adults of the same age [7]. In particular, a loss of muscle strength in the ankle dorsiflexion muscles of stroke patients causes an inefficient swing phase of walking, which reduces the walking speed $[8,9]$. Therefore, strengthening exercise to recover the lost muscle strength of stroke patients must be included in programs to improve walking ability [10]. In some cases of chronic stroke patients, however, strength strengthening exercise is difficult to apply due to the severe muscle weakness and psychological stress on the lower extremity [9,11].

Cross-training defines that motor activity in the unilateral limbs affects the performance of the homologous muscles of the contralateral limbs [12-15]. The mechanism of cross-training has not been elucidated thus far, but it is explained by the neural adaptation of unilateral limb activity [16]. Sun et al. [17] applied high-intensity isometric resistance training to the unaffected wrist extensors for five weeks to examine the effects of cross-training on the paralyzed upper extremities. Kim et al. [18] reported the results of a three-week progressive task-oriented training of the non-paralytic lower limbs to improve the muscle strength in paralyzed lower limbs. On the other hand, previous studies on the effectiveness of cross-training focusing on strengthening exercise in the non-paralytic lower extremities in chronic stroke patients have been insufficient. Therefore, this study examined the effects of bilateral ankle dorsiflexion muscle-strength exercise using cross-training on chronic stroke patients. This study hypothesized that bilateral ankle dorsiflexion musclestrengthening exercise would be more effective in improving the function of stroke patients than paraplegic dorsiflexion muscle strengthening exercise.

\section{Methods}

\section{Subjects}

The subjects were chronic adult hemiplegic patients who were diagnosed with stroke and were hospitalized at the D rehabilitation hospital. The criteria for selection of the subjects were as follows: those six months or more after being diagnosed with a stroke, could walk more than 10 $\mathrm{m}$ independently without assistive devices indoors, had an ankle joint manual movement range of five degrees or more with the knee joint extended, and had a score of 24 or higher on the Mini-Mental Status Examination-Korean version (MMSE-K). The exclusion criteria were those with a history of orthopedic injuries that may affect walking, visual or auditory deficits, severe cardiovascular problems, and below Trace in the ankle joint anterior muscle strength test. Of the 81 patients with chronic stroke, who were undergoing rehabilitation at D rehabilitation hospital, 24 patients met the inclusion and exclusion criteria. The 24 subjects were informed of the purpose and method of the study and agreed to participate in the study. This study was conducted after gaining approval of the Institutional Bioethics Committee of Daejeon University (IRB: 1040647-201506-HR-011-03). A total of 24 subjects were assigned randomly to the bilateral ankle dorsiflexion muscle-strengthening training group (experimental group $(\mathrm{n}=12))$ and the paraplegic ankle dorsiflexion muscle strengthening training group (control group $(n=12)$ ) 


\section{Intervention}

All 24 subjects selected underwent general neurodevelopmental rehabilitation therapy five times a week for 30 minutes per session, for six weeks. In addition, the experimental group performed bilateral ankle dorsiflexion muscle-strengthening training three times a week, 30 minutes per session, for six weeks. The control group performed the paraplegic ankle dorsiflexion muscle-strengthening training in the same manner.

Ankle dorsiflexion muscle-strengthening training consisted of warm-up, main, and cool down exercises [19]. The warm-up exercise was performed for five minutes to promote joint movement and improve the awareness of movement in the supine position [20]. The main exercise followed the exercise suggested by Bird et al. [21]. Bilateral ankle dorsiflexion muscle-strengthening exercise was applied for five sets for 30 minutes with 10 repetitions set as a single set; a three to five minute rest period was provided between each set. The type of muscle contraction and contraction time applied to the strengthening exercise consisted of two seconds of concentric contraction, two seconds of isometric contraction, and two seconds of eccentric contraction. To perform the exercise, the non-paralytic ankle dorsiflexion muscle strengthening exercise was performed, and the paraplegic ankle dorsiflexion muscle strengthening exercise was then carried out alternately on one set of each. A physiotherapist, who had no information on the study, supervised the muscle-strengthening exercise at the fifth year of the clinical trial. The strength of the power resistance was set to the intensity (10 RM) at which the subject can repeat the operation for up to 10 times. The application of resistance started with the subject's toes and then applied $1 / 3$ of the foot or the head of the metatarsal bone [22]. The strengthening exercise was applied to the paraplegic ankle joint so that the therapist could feel the muscle contraction of the ankle dorsiflexior during ankle joint flexion, and the therapist fully supported the paralyzed ankle joint. All other procedures were applied in the same manner as non-paralytic strength training. Difficulties in exercise performance were in the supine position at week one to two, sitting position at weeks three to four, and in the standing position at weeks five to six [11]. In the control group, the strengthening exercise was performed only on the paralyzed dorsiflexion muscle in the same manner as the paralytic ankle dorsiflexion muscle-strengthening exercise of the experimental group.

\section{Outcome measure}

Muscle activity of paralytic tibialis anterior

Surface electromyography QEMG-4 (Laxtha Inc., Daejeon, Korea) and TeleScan ver 3.28 (Laxtha Inc., Daejeon, Korea) software were used to evaluate the muscle activity of the paralytic tibialis anterior muscle. To collect the EMG signals, the epidermis was sterilized with an epilation of the skin area and an alcohol swab, and a disposable anode electrode $(\mathrm{Ag} / \mathrm{AgCl})$ was attached. The electrode attachment site of the anterior tibialis was $2 / 3$ of the distance from the lateral condyle of the tibia to the first cuneiform bone [23]. The active electrode was attached at a distance of $1.5 \mathrm{~cm}$ in parallel to the direction of the muscle fibers of the tibialis anterior. The reference electrode was also attached to the front of the patellar bone. The EMG signal was set to a sampling rate of $1024 \mathrm{~Hz}$, and notch filtering for removing the signal noise was 60 Hz. The frequency bandwidth (band-pass filtering) was set to 20-500 $\mathrm{Hz}$. To measure the maximum voluntary isometric contraction (MVIC) of the paraplegic tibialis anterior of the subject, the posture and procedure of the manual strength assessment presented by Kendall et al. [22] were performed. The raw EMG signal collected during five seconds of MVIC was the RMS (root mean square) processed for three seconds except for one second at the beginning and end, and the average value of the total measured three times was calculated. A break of 30 seconds was provided between each measurement. The $\%$ RVC 
(reference voluntary contraction) method was used to standardize the calculated MVIC [24]. RVC EMG signal measured the muscle activity of the paralytic tibialis anterior while the subject remained static standing for five seconds. The measured RVC EMG signal obtained the RMS signal with three seconds of data except for one second at the beginning and end, and the average value of the measured three times was calculated.

\section{1) Balancing ability}

A Timed Up and Go Test (TUG) was performed to assess the subject's ability to balance. The TUG test measured the time it took to sit on a chair with armrests, listen to the signal, and stand up to walk three meters back. This tool is useful for stroke hemiplegic patients, and the validity and reliability are .99 [25]. Three measurements were taken, and the average used for data analysis.

\section{2) Gait function}

The $10 \mathrm{~m}$ walking speed test (10 MWT) was performed to evaluate the gait function of the subjects. To provide the distance for the acceleration and deceleration of walking, the 10 MWT marked the start point and end point on the floor by adding an additional $2 \mathrm{~m}$ from the $10 \mathrm{~m}$ distance [26]. The start time of the measurement was recorded by dividing the walking speed by one-hundredth of a second using a stopwatch. The walking speed was measured at a comfortable speed. One preliminary trial was made before the measurement, and the average of three measurements was used. This test had high reliability of .95 and a tester of .90 [27].

\section{Data analysis}

Data collected in this study were processed statistically using SPSS version 25.0 for Windows. A Shapiro-Wilk test was conducted to test the normality of all data, and the general characteristics of the subjects were tested using a chi-square test and independent sample $t$ test. A Wilcoxon signed rank test was performed to verify the statistical significance of the tibialis anterior muscle activity, balance ability, and gait function before and after the intervention within each group. A Mann-Whitney's U-test was used to compare the changes before and after the intervention between the two groups. The statistical significance level was set to $a=.05$.

\section{Results}

Twenty-four subjects participated in this study, but five dropped out (three in the experimental group and two in the control group) due to discharge. Table 1 lists the general characteristics of the 19 subjects (nine in the experimental groups and 10 in the control groups) who completed the study. In the weights of the two groups, the experimental group had an average of $9 \mathrm{~kg}$ more than the control group $(\mathrm{p}<.05)$. On the other hand, no significant differences in gender, age, height, paralytic side, injury factor, and onset duration of the disease were observed between the two groups $(\mathrm{p}>.05)$.

Table 2 lists the muscle activity of the paralytic tibialis anterior, TUG, and 10 MWT before and after intervention in the experimental and control groups. Both groups showed significant differences in post training muscle activity, TUG, and 10MWT compared to pretreatment ( $<<.05)$. The muscle activity of the paralytic tibialis anterior was higher after training than before training (experimental group, $740 \%$ of MVIC vs. $1699 \%$ of MVIC; control group, $1029 \%$ of MVIC vs. $1735 \%$ of MVIC, respectively) ( $<<.05)$. The TUG after training was lower than before training (experimental group, $20.7 \mathrm{~m} / \mathrm{sec}$ vs. $14.0 \mathrm{~m} / \mathrm{s}$; control group, $36.8 \mathrm{~m} / \mathrm{sec}$ vs. $28.3 \mathrm{~m} / \mathrm{sec}$, respectively) $(\mathrm{p}<.05)$. The 10MWT after training was lower than before training (experimental group, $18.9 \mathrm{~m} / \mathrm{sec}$ vs. $14.1 \mathrm{~m} / \mathrm{s}$; control group, $36.3 \mathrm{~m} / \mathrm{sec}$ vs. $28.8 \mathrm{~m} / \mathrm{sec}$, respectively) ( $<<.05)$. On the other hand, there were no significant differences 
Table 1. General Characteristics of the Subjects

\begin{tabular}{cccc}
\hline & $\begin{array}{c}\text { Experimental Group } \\
(\mathrm{n}=9)\end{array}$ & $\begin{array}{c}\text { Control Group } \\
(\mathrm{n}=10)\end{array}$ & $p$-value \\
\hline Sex (male/female) & $5 / 4$ & $4 / 6$ & .656 \\
Affected Side (right/left) & $6 / 3$ & $5 / 5$ & .650 \\
Factor (infarction/hemorrhage) & $7 / 2$ & $7 / 3$ & 1.000 \\
Height (cm) & $160.6 \pm 7.3$ & $158.30 \pm 8.2$ & .536 \\
Weight (kg) & $66.1 \pm 5.1$ & $56.90 \pm 10.5$ & .029 \\
Onset Time (months) & $16.3 \pm 15.9$ & $24.1 \pm 17.6$ & .328 \\
Age (years) & $67.6 \pm 7.3$ & $65.60 \pm 6.7$ & .551 \\
\hline
\end{tabular}

Values are expressed as means \pm standard deviations or numbers.

Table 2. Comparison of EMG and Gait Capacity Before and After Training within Each Group and Between the Two Groups

\begin{tabular}{|c|c|c|c|c|}
\hline & & $\begin{array}{l}\text { Experimental Group } \\
\qquad(\mathrm{n}=9)\end{array}$ & $\begin{array}{l}\text { Control Group } \\
\qquad(\mathrm{n}=10)\end{array}$ & $Z$ \\
\hline \multirow{4}{*}{ EMG ( $\%$ of RVC) } & Pre & $740.78 \pm 364.51$ & $1029.65 \pm 1267.26$ & -.735 \\
\hline & Post & $1699.13 \pm 1248.22$ & $1735.54 \pm 1396.44$ & -.163 \\
\hline & Z & $-2.310^{*}$ & $-1.886^{*}$ & \\
\hline & Change & $958.35 \pm 1078.55$ & $705.90 \pm 1168.32$ & -.082 \\
\hline \multirow{4}{*}{ TUG $(\mathrm{m} / \mathrm{sec})$} & Pre & $20.67 \pm 8.71$ & $36.79 \pm 31.55$ & -.980 \\
\hline & Post & $14.00 \pm 3.99$ & $28.28 \pm 22.82$ & -1.551 \\
\hline & Z & $-2.666^{*}$ & $-2.701^{*}$ & \\
\hline & Change & $-6.67 \pm 5.38$ & $-8.51 \pm 10.27$ & -.245 \\
\hline \multirow{4}{*}{ 10MWT (m/sec) } & Pre & $18.92 \pm 8.90$ & $36.33 \pm 35.58$ & -1.144 \\
\hline & Post & $14.05 \pm 5.34$ & $28.76 \pm 25.30$ & -1.388 \\
\hline & Z & $-2.547^{*}$ & $-2.497^{*}$ & \\
\hline & Change & $-4.87 \pm 4.59$ & $-7.57 \pm 11.16$ & .000 \\
\hline
\end{tabular}

Values are expressed as means \pm standard deviations

EMG, electromyography; 10 MWT, $10 \mathrm{~m}$ walking test; TUG, timed up and go. ${ }^{*} p<.05$

in the changes before and after the intervention between the two groups.

\section{Discussion}

This study examined the effects of cross-training by comparing the bilateral dorsiflexion muscle strengthening exercise and paralytic dorsiflexion muscle-strengthening exercise in patients with chronic stroke. As a result, significant improvements in the muscle activity, balance ability, and walking ability were observed after the intervention in both the experimental and control groups, but there was no significant difference between the groups. These results were different from the hypothesis in this 
study that bilateral dorsiflexion muscle strengthening exercise would be more effective, but the improvement of muscle activity of the paralytic tibialis anterior muscle before and after intervention in the experimental group proved the positive effect of cross-training. These results were attributed partly to the muscles of the lower extremity also being involved in muscle contraction to perform the training during non-paralytic flexion muscle strengthening exercise $[12,15]$.

Dragert et al. [9] conducted isometric resistance training on the non-paralytic dorsiflexion muscles for six weeks in patients with chronic stroke. As a result, the muscle activity of the paralytic tibialis anterior was increased. These findings are in part, consistent with those in the experimental group. Song [11] reported the effects of cross-training on the psychological states in stroke patients and found that non-paralyzed resistance training was more effective in reducing depression than paralyzed resistance training. This supports the necessity of an approach to strengthening muscles toward non-paralysis. In addition, when considering the aspects that muscle strength of the ankle dorsiflexor muscle plays an important role in the walking ability, such as walking speed and walking endurance [8,28,29], paralyzed and non-paralyzed ankle dorsiflexion muscle-strengthening exercise had positive effects on increasing the walking velocity in both groups. Moon \& Kim [30] reported that in patients with chronic stroke, gradual strengthening exercise on the lower extremity in the open chain was more effective in reducing the TUG time than the closed chain. In this study, the TUG time decreased after open chain motion in the ankle dorsiflexion muscle, showing similar results.

Jeon and Hwang [31] compared the gait function after strengthening training in the paralytic and non-paralytic hip extension muscles of stroke patients. In both groups, there was a significant increase in walking speed after the intervention, but there was no difference between the two groups. These results suggest that the application of interventions to the non-paralyzed side, in addition to the paralyzed side, may be an effective treatment.

Weakened ankle joint dorsiflexor muscles in stroke patients are a major factor causing difficulty in lifting toes during walking, which is a major factor causing circumduction gait [8]. Therefore, this study focused on strengthening the ankle dorsiflexion muscle. As a result, the muscle activity of the tibialis anterior muscle after the interventions was increased significantly in the experimental and control group compared to that before the intervention. Therefore, the strengthening training method performed in this study is an effective intervention method for improving the muscle strength of the paralytic ankle dorsiflexion muscle. Sousa et al. [32] suggested that to start walking in a standing position, postural stability should be secured by controlling the activity of the tibialis anterior and the inhibition of the soleus muscle. In terms of walking speed, it was more effective to use an ankle joint strategy than the hip joint strategy $[8,29]$. Therefore, the improvement of the muscle activity of paralytic tibialis anterior in this study appears to be of high clinical value for the improvement of gait function in stroke patients.

In this study, a significant difference in body weight was observed between the two groups. On the other hand, no significant differences in terms of gender, injury factors, and height, which could affect the group homogeneity in addition to body weight, were observed between the two groups. Nevertheless, this pilot study has limitations in generalizing the results of this study. The relatively short duration of the intervention (six weeks) and the lack of follow-up after the end of the intervention is a factor limiting the generalization of the findings to patients with chronic stroke. On the other hand, this study is significant because it clinically examined the cross-training effects of absolute ankle dorsiflexion strengthening exercise in the functional recovery of chronic stroke patients. Although further studies will be needed to overcome the limitations in this study, these results suggest a new intervention 
method that can be applied to stroke patients who are unable to apply strengthening exercise to the paralytic lower extremities.

\section{Conclusion}

This study examined the effects of cross-training as a strengthening exercise in chronic stroke patients. The results revealed a similar effect in both the muscle strengthening exercise applied to the paralyzed side as well as the non-paralyzed side. Therefore, bilateral ankle dorsiflexion muscle-strengthening exercise may be an alternative to stroke patients who have limitations in applying the muscle strengthening exercise only to the paralytic lower extremities.

\section{References}

[1] Feigin VL, Krishnamurthi RV, Parmar P, et al. Update on the Global Burden of Ischemic and Hemorrhagic Stroke in 1990-2013: The GBD 2013 Study. Neuroepidemiology. 2015;45(3):161-76.

[2] Tyson SF, Hanley M, Chillala J, et al. Balance disability after stroke. Phys Ther. 2006;86(1):30-8.

[3] Hendrickson J, Patterson KK, Inness EL, et al. Relationship between asymmetry of quiet standing balance control and walking post-stroke. Gait Posture. 2014;39(1):177-81.

[4] Sprigg N, Selby J, Fox L, et al. Very low quality of life after acute stroke: data from the efficacy of nitric oxide in stroke trial. Stroke. 2013;44(12):3458-62.

[5] Kim DG, Choi YR, Cha YJ. Comparison of exercise intensity of strengthening exercise program for stroke patients with type II diabetes melitus. J Korean Soc Phys Med. 2018;13(4):105-12.

[6] Park JH, Lee SG. Effect of bilateral arm movement on brain and muscle activity in chronic stroke patients. J Korean Soc Phys Med. 2018;13(1):1-9.

[7] Dorsch S, Ada L, Canning CG. Lower Limb Strength Is Significantly Impaired in All Muscle Groups in
Ambulatory People With Chronic Stroke: A CrossSectional Study. Arch Phys Med Rehabil. 2016;97(4): 522-7.

[8] Dorsch S, Ada L, Canning CG, et al. The strength of the ankle dorsiflexors has a significant contribution to walking speed in people who can walk independently after stroke: an observational study. Arch Phys Med Rehabil. 2012;93(6):1072-6.

[9] Dragert K, Zehr EP. High-intensity unilateral dorsiflexor resistance training results in bilateral neuromuscular plasticity after stroke. Exp Brain Res. 2013;225(1):93-104.

[10] Moreland JD, Goldsmith CH, Huijbregts MP, et al. Progressive resistance strengthening exercises after stroke: a single-blind randomized controlled trial. Arch Phys Med Rehabil. 2003;84(10):1433-40.

[11] Song GB. Effects of indirect cross training on strengthening, balance, gait and depression in patients with stroke. Doctor's Degree. Daegu. 2015.

[12] Yasuda Y, Miyamura M. Cross transfer effects of muscular training on blood flow in the ipsilateral and contralateral forearms. Eur J Appl Physiol Occup Physiol. 1983; 51(3):321-9.

[13] Stromberg BV. Contralateral therapy in upper extremity rehabilitation. Am J Phys Med. 1986;65(3):135-43.

[14] Hortobagyi T, Scott K, Lambert J, et al. Cross-education of muscle strength is greater with stimulated than voluntary contractions. Motor Control. 1999;3(2):205-19.

[15] Zhou S. Chronic neural adaptations to unilateral exercise: mechanisms of cross education. Exerc Sport Sci Rev. 2000;28(4):177-84.

[16] Lee M, Carroll TJ. Cross education: possible mechanisms for the contralateral effects of unilateral resistance training. Sports Med. 2007;37(1):1-14.

[17] Sun Y, Ledwell NMH, Boyd LA, et al. Unilateral wrist extension training after stroke improves strength and neural plasticity in both arms. Exp Brain Res. 2018;236(7): 2009-21.

[18] Kim CY, Lee JS, Kim HD, et al. The effect of progressive 
task-oriented training on a supplementary tilt table on lower extremity muscle strength and gait recovery in patients with hemiplegic stroke. Gait Posture. 2015;41(2): 425-30

[19] Ramas J, Courbon A, Roche F, et al. Effect of training programs and exercise in adult stroke patients: literature review. Ann Readapt Med Phys. 2007;50(6):438-44, 0-7.

[20] Kisner C, Colby LA. Therapeutic exercise: foundations and techniques Philadelphia. F. A. Davis Company. 2013.

[21] Bird SP, Tarpenning KM, Marino FE. Designing resistance training programmes to enhance muscular fitness: a review of the acute programme variables. Sports Med. 2005;35(10):841-51.

[22] Kendall FP, McCreary EK, Provance PG. Muscles testing and function, with posture and pain. Baltimore. Lippincott Williams \& Wilkins. 2005.

[23] Criswell E. Cram's introduction to surface electromyography. Sudbury. :Jones \& Bartlett Publishers. 2010.

[24] Lehman GJ, McGill SM. The importance of normalization in the interpretation of surface electromyography: a proof of principle. J Manipulative Physiol Ther. 1999;22(7): 444-6.

[25] Ng SS, Hui-Chan CW. The timed up \& go test: its reliability and association with lower-limb impairments and locomotor capacities in people with chronic stroke. Arch
Phys Med Rehabil. 2005;86(8):1641-7.

[26] Geroin C, Picelli A, Munari D, et al. Combined transcranial direct current stimulation and robot-assisted gait training in patients with chronic stroke: a preliminary comparison. Clin Rehabil. 2011;25(6):537-48.

[27] Mehrholz J, Wagner K, Rutte K, et al. Predictive validity and responsiveness of the functional ambulation category in hemiparetic patients after stroke. Arch Phys Med Rehabil. 2007;88(10):1314-9.

[28] Ng SS, Hui-Chan CW. Ankle dorsiflexor, not plantarflexor strength, predicts the functional mobility of people with spastic hemiplegia. J Rehabil Med. 2013;45(6):541-5.

[29] Mentiplay BF, Adair B, Bower KJ, et al. Associations between lower limb strength and gait velocity following stroke: a systematic review. Brain Inj. 2015;29(4):409-22.

[30] Moon SH, Kim YM. Effects of close kinetic chain resistant exercise of lower extremity on the gait with stroke. J Korean Soc Phys Med. 2014;9(4):475-83.

[31] Jeon HJ, Hwang BY. Effect of bilateral lower limb strengthening exercise on balance and walking in hemiparetic patients after stroke: a randomized controlled trial. J Phys Ther Sci. 2018;30(2):277-81.

[32] Sousa AS, Silva A, Santos R. Ankle anticipatory postural adjustments during gait initiation in healthy and post-stroke subjects. Clin Biomech (Bristol, Avon). 2015;30(9):960-5. 